\title{
Plasma level of hydroxysteroid (17- $\beta$ ) dehydrogenase 1 in the second trimester is an independent risk factor for predicting preeclampsia after adjusting for the effects of mean blood pressure, bilateral notching and plasma level of soluble fms-like tyrosine kinase $1 /$ placental growth factor ratio
}

\author{
Akihide Ohkuchi ${ }^{1,3}$, Osamu Ishibashi ${ }^{2,3}$, Chikako Hirashima ${ }^{1}$, Kayo Takahashi ${ }^{1}$, Shigeki Matsubara ${ }^{1}$, \\ Toshihiro Takizawa ${ }^{2}$ and Mitsuaki Suzuki ${ }^{1}$
}

\begin{abstract}
Mean blood pressure (MBP), bilateral notching (BN) in the uterine artery and increased circulating levels of soluble fms-like tyrosine kinase-1/placental growth factor (sFlt-1/PIGF) ratio are predictors of preeclampsia (PE). Recently, we disclosed that reducing the plasma level of hydroxysteroid (17- $\beta$ ) dehydrogenase 1 (HSD17B1), which is a steroidogenetic enzyme catalyzing the conversion of estrone to $17 \beta$-estradiol, is a potential prognostic factor for PE. Our aim was to evaluate whether HSD17B1 is an independent risk factor for predicting PE after adjusting for the effects of MBP, BN and the plasma level of the sFlt-1/PIGF ratio in the second trimester. One hundred and twenty-eight consecutive normal pregnant women without gestational hypertension (GH) or PE and 30 women with PE were selected from 1724 pregnant women. Multivariate logistic regression with a forward stepwise procedure was used to construct a prediction model. A past history of GH/PE, a family history of hypertension, pre-pregnancy body mass index, MBP, BN, plasma levels of sFIt-1/PIGF ratio and plasma levels of HSD17B1 were significantly associated with the occurrence of PE; however, only MBP (OR (95\% confidence interval), 1.08 (1.03-1.14)), BN (7.5 (1.9-30)), sFlt-1/PIGF (21 (2.7-163)) and HSD17B1 $(0.43(0.22-0.85))$ were independent risk factors for PE. The area under the receiver-operating-characteristic curve for the combination model was 0.89 , yielding a sensitivity of 0.84 , a specificity of 0.88 and a positive likelihood ratio of $7.2(4.0-13)$. In conclusion, HSD17B1 is an independent risk factor for $\mathrm{PE}$, and the combination of several risk factors including HSD17B1 in the second trimester may improve the prediction of PE. Hypertension Research (2012) 35, 1152-1158; doi:10.1038/hr.2012.109; published online 12 July 2012
\end{abstract}

Keywords: bilateral notching; HSD17B1; mean blood pressure; preeclampsia; sFlt-1

\section{INTRODUCTION}

The well-known risk factors for preeclampsia (PE) are primiparity, ${ }^{1,2}$ pregnancy in elderly women, ${ }^{1,3}$ a previous history of $\mathrm{PE}$ or gestational hypertension (GH), ${ }^{2,3}$ a family history of hypertension, ${ }^{3,4}$ high prepregnancy body mass index (BMI), ${ }^{1,3,5}$ blood pressure levels, ${ }^{1,3,5}$ bilateral notching (BN) in the uterine artery, ${ }^{3,6}$ and increased circulating levels of the soluble fms-like tyrosine kinase-1/placental growth factor (sFlt-1/PlGF) ratio., ${ }^{3,7}$ Recently, we disclosed that a decreased plasma level of hydroxysteroid (17- $\beta$ ) dehydrogenase 1
(HSD17B1) precedes the onset of $\mathrm{PE}$ and is a new potential prognostic factor for PE. ${ }^{8}$ HSD17B1 is a steroidogenic enzyme catalyzing the conversion of estrone to $17 \beta$-estradiol and is dysregulated by $m i R-210$ and $m i R-518 c$, which are aberrantly expressed in preeclamptic placenta. ${ }^{8}$ In a previous study, plasma HSD17B1 protein levels were found to be decreased in women with both early-onset PE (EO-PE) and late-onset PE (LO-PE), and plasma HDS17B1 levels were also found to be decreased in pregnant women at $20-23$ and $27-30$ weeks of gestation in whom PE occurred later. ${ }^{8}$

${ }^{1}$ Department of Obstetrics and Gynecology, Jichi Medical University School of Medicine, Tochigi, Japan and ${ }^{2}$ Department of Molecular Medicine and Anatomy, Nippon Medical School, Tokyo, Japan

${ }^{3}$ These authors contributed equally to this work

Correspondence: Dr A Ohkuchi, Department of Obstetrics and Gynecology, Jichi Medical University School of Medicine, 3311-1 Yakushiji, Shimotsuke-shi, Tochigi 329-0498, Japan.

E-mail: okuchi@jichi.ac.jp

Received 20 March 2012; revised 8 May 2012; accepted 10 May 2012; published online 12 July 2012 
However, in the previous study, we did not investigate whether there is a significant correlation between plasma levels of HSD17B1 and angiogenic/antiangiogenic factors. Hence, the combined use of HSD17B1 and other factors may be further advantageous for clinical use. In the current study, our aim was to evaluate whether HSD17B1 in the second trimester is an independent risk factor for predicting all $\mathrm{PE}$, after adjusting for the effects of potential risk factors for $\mathrm{PE}$, including blood pressure levels, $\mathrm{BN}$ and plasma levels of the sFlt-1/PlGF ratio.

\section{METHODS}

\section{Subjects and procedures}

We obtained written informed consent from all women as well as approval from the Ethics Committee of our institute. The study subjects consisted of two groups: 128 consecutive normal pregnant women without GH or PE, and 30 preeclamptic women before the onset of PE. The study subjects were all Japanese women with a singleton pregnancy. Blood samples from normal pregnant women were collected at 19-25 weeks of gestation; the samples were collected between December 2006 and September 2008. Blood samples from preeclamptic women were collected at 19-25 weeks of gestation in a prospective cohort study between April 2004 and September 2008. These samples were used in our previous study. ${ }^{8}$

Blood samples were collected into tubes containing EDTA, centrifuged at $4{ }^{\circ} \mathrm{C}$ at 2500 r.p.m. for $15 \mathrm{~min}$, and stored at $-20^{\circ} \mathrm{C}$ until use. Electrochemiluminescence immunoassays of sFlt-1 and PlGF using Elecsys sFlt-1 and Elecsys PlGF were performed using Modular Analytics E 170 (Roche Diagnostics, Mannheim, Germany) according to the manufacturer's instructions. The minimal detectable doses in the assays for serum sFlt-1 and PlGF were 15 and $10 \mathrm{pg} \mathrm{ml}^{-1}$, respectively; the intraassay and interassay coefficients of variation for serum sFlt-1 were $0.5-6.8 \%$ and $0.7-11 \%$, respectively; the intraassay and interassay coefficients of variation for serum PlGF were $0.6-2.6 \%$ and $0.6-5.9 \%$, respectively (manufacturer's information). The plasma recovery rates of sFlt-1 and PlGF were 0.89 and 0.85 , respectively. ${ }^{9}$ We used the data of plasma levels of HSD17B1, which had been measured by sandwich ELISA (enzyme-linked immunosorbent assay) in our previous study. ${ }^{8}$

\section{Measurements of $\mathrm{BN}$ in the uterine artery}

Color-pulsed Doppler ultrasound examination was performed once at 20-23 weeks of gestation. At least three waveforms of the uterine artery from each side were viewed on the monitor. We defined notching as positive when a notch was seen in all three consecutive waveforms, and $\mathrm{BN}$ as positive when notches were seen in both uterine arteries.

\section{Blood pressure in pregnancy and puerperal period}

Blood pressure was measured with a validated Omron HEM-906 automated digital oscillometric sphygmanometer (OMRON Healthcare, Kyoto, Japan), according to standard procedures. The mean value of two blood pressure readings with at least a one-day interval at 16-23 weeks of gestation was documented for each participant.

\section{Definitions of PE and a small-for-gestational-age infant}

We defined PE according to the definition and classification of PIH (2004) of the Japan Society for the Study of Hypertension in Pregnancy (JSSHP). ${ }^{10}$ In brief, PE was defined as hypertension with proteinuria occurring after 20 weeks of gestation. Superimposed PE was defined as chronic hypertension diagnosed before pregnancy or before 20 weeks of gestation, with proteinuria emerging afterward. Superimposed PE was included in the category of PE in this study. Hypertension was defined as systolic blood pressure $\geqslant 140 \mathrm{~mm} \mathrm{Hg}$ and/or diastolic blood pressure $\geqslant 90 \mathrm{~mm} \mathrm{Hg}$ on 2 occasions $\geqslant 4 \mathrm{~h}$ apart. Proteinuria was defined as $\geqslant 300 \mathrm{mg}$ per day from 24 -h urine collection. If only test tape was available, repeated semi-quantitative test results of $1+$, which represented $30 \mathrm{mg} \mathrm{dl}^{-1}$ protein or more, were considered to constitute a positive result. We defined PE with onset earlier than 32 weeks of gestation as EO-PE, and onset at 32 weeks of gestation or later as LO-PE, according to the definition of
JSSHP. ${ }^{10}$ It should be noted that EO-PE has been commonly defined as PE with onset at $<34$ weeks of gestation in the previous reports. ${ }^{11-13}$ We defined a small-for-gestational-age infant as having a birth weight below the 10th percentile for the Japanese population. ${ }^{14}$

\section{Statistics}

The unpaired $t$-test was used for continuous variables. The $\chi^{2}$-test or Fisher's exact test was used for categorical data. The area under the receiver-operatingcharacteristic curves (AROCs) and 95\% confidence intervals (CIs) for predicting PE were calculated using SPSS software package (version 13.0J for Windows; SPSS Inc, Chicago, IL, USA). A prediction model with multiple variables was constructed using a forward stepwise procedure in logistic regression analysis in the SPSS. The statistical levels of significance determined with the log-likelihood ratio test for inclusion and exclusion were set at $P=0.05$ and $P=0.10$, respectively. The positive likelihood ratio $(\mathrm{LR}+)$ and the 95\% CIs were calculated using the Confidence Interval Calculator (created by Rob Herbert in The University of Sydney). $P<0.05$ was considered significant.

\section{RESULTS}

In the preeclamptic group, significantly more women had a past history of GH/PE, a family history of hypertension and BN at 20-23 weeks of gestation than in the normal pregnant group. Additionally, in preeclamptic women, pre-pregnancy BMI and mean blood pressure (MBP) were significantly higher at 16-23 weeks of gestation than in controls, and gestational age at delivery and birth weight were significantly lower (Table 1 ).

When we evaluated the AROCs of various potential risk factors for predicting all $\mathrm{PE}$, a past history of $\mathrm{GH} / \mathrm{PE}$, a family history of hypertension, pre-pregnancy BMI and MBP at 16-23 weeks of gestation, $\mathrm{BN}$ at $20-23$ weeks of gestation, plasma levels of the sFlt1/PlGF ratio at 19-25 weeks of gestation and plasma levels of HSD17B1 at 19-25 weeks of gestation were significant factors (Table 2 and Figure 1). The best multivariate model was constructed using a forward stepwise procedure in logistic regression analysis including the above mentioned significant variables, revealing that MBP at 16-23 weeks, BN, plasma levels of the sFlt-1/PlGF ratio and plasma levels of HSD17B1 were independent risk factors for predicting all PE (Table 3). The addition of BN to the model of only MBP changed the positive likelihood ratio $(\mathrm{LR}+)$ from 3.1 to 3.7 ; the addition of the sFlt-1/PlGF ratio to the model involving both MBP and $\mathrm{BN}$ changed the $\mathrm{LR}+$ to 5.0; and the addition of HSD17B1 to the model involving MBP, BN and the sFlt-1/PlGF ratio together also changed the LR + to 7.2 (Table 2 and Figure 1). The adjusted odds ratios for these four markers were almost equal to the crude odds ratios, suggesting that the interactions of these four markers were very weak. There were no correlations between HSD17B1 and the sFlt-1/PlGF ratio in preeclamptic women and normal pregnant women (Spearman's correlation coefficients: $0.220(P=0.291)$ and $0.093(P=0.361)$, respectively). The combination model of MBP, BN, the sFlt-1/PlGF ratio and HSD17B1 yielded an AROC of 0.89 , a sensitivity of 0.84 , a specificity of 0.88 and an LR $+(95 \% \mathrm{CI})$ of $7.2(4.0-13)$.

Although we did not perform multivariate analysis for evaluating various risk factors on LO-PE or EO-PE owing to the relatively small sample size, we simply compared risk factors in normal pregnant women, women with LO-PE and women with EO-PE (Table 4). In women with LO-PE, the proportion of women with a past history of $\mathrm{GH} / \mathrm{PE}$, a pre-pregnancy $\mathrm{BMI} \geqslant 24$, an $\mathrm{MBP} \geqslant 91.3 \mathrm{~mm} \mathrm{Hg}$, a $\mathrm{BN}$, a sFlt-1/PlGF ratio $\geqslant 6.9$ and HSD17B1 $<43 \mathrm{ng} \mathrm{ml}^{-1}$ was significantly higher than that for the normal pregnant group. In women with EO-PE, the proportion of women with a family history of 
Table 1 Maternal and neonatal characteristics in normal pregnant women and preeclamptic women

\begin{tabular}{|c|c|c|c|c|}
\hline Characteristics & $\begin{array}{l}\text { Normal pregnant women } \\
\qquad(\mathrm{n}=128)^{\mathrm{a}}\end{array}$ & $\begin{array}{l}\text { All preeclamptic women } \\
\qquad(\mathrm{n}=30)\end{array}$ & $\begin{array}{l}\text { Numbers of } \\
\text { missing values }\end{array}$ & P-value \\
\hline \multicolumn{5}{|l|}{ Mother } \\
\hline Age $\geqslant 35$ years $(\%)$ & $43(34)$ & $13(43)$ & 0 & 0.397 \\
\hline Race: Japanese (\%) & $127(99.2)$ & $30(100)$ & 0 & 1.000 \\
\hline Nulliparous women (\%) & $65(51)$ & $14(47)$ & 0 & 0.840 \\
\hline Current smoker (\%) & $7(5.7)$ & $14(13)$ & 6 & 0.229 \\
\hline Pre-pregnancy BMI (mean \pm s.d., $\mathrm{kg} \mathrm{m}^{-2}$ ) & $22.2 \pm 4.1$ & $24.2 \pm 4.4$ & 3 & 0.021 \\
\hline Obesity (\%) & $27(21)$ & $11(39)$ & 3 & 0.054 \\
\hline Chronic hypertension (\%) & $0(0.0)$ & $6(20)$ & 0 & $<0.001$ \\
\hline MBP at $16-23$ weeks of gestation (mean \pm s.d., $\mathrm{mm} \mathrm{Hg}$ ) & $86 \pm 10$ & $99 \pm 14$ & 0 & $<0.001$ \\
\hline MBP at 26-31 weeks of gestation (mean \pm s.d., $\mathrm{mm} \mathrm{Hg}$ ) & $84 \pm 9$ & $100 \pm 14$ & 1 & $<0.001$ \\
\hline \multicolumn{5}{|l|}{ Infant } \\
\hline Preterm delivery (\%) & $5(3.9)$ & $12(40)$ & 0 & $<0.001$ \\
\hline Birthweight (mean \pm s.d., g) & $3001 \pm 362$ & $2564 \pm 791$ & 0 & 0.006 \\
\hline Multiple of median of birthweight $(\text { mean } \pm \text { s.d. })^{b}$ & $-0.03 \pm 0.11$ & $-0.05 \pm 0.16$ & 0 & 0.474 \\
\hline Small-for-gestational-age infant $(\%)^{c}$ & $14(11)$ & $6(20)$ & 0 & 0.220 \\
\hline
\end{tabular}

Abbreviations: BMI, body mass index; BN, bilateral notching; GH, gestational hypertension; MBP, mean blood pressure; PE, preeclampsia.

a Normal pregnant women were defined as women not complicated with PE and GH.

${ }^{b}$ Multiple of median of birthweight was calculated by the equation of (birthweight-median)/median, where median was defined by standard birth weight table for the Japanese population.

'Small-for-gestational-age infant was defined as an infant having a birth weight below the 10th percentile for the Japanese population.

Table 2 Screening performances of possible risk factors for predicting all PE in the second trimester

\begin{tabular}{|c|c|c|c|c|c|}
\hline \multirow[b]{2}{*}{ Risk factors } & \multicolumn{5}{|c|}{ All preeclamptic women } \\
\hline & AROC $(95 \% \mathrm{Cl})$ & Cutoff & $S E$ & $S P$ & $L R+(95 \% \mathrm{Cl})$ \\
\hline \multicolumn{6}{|l|}{ Maternal characteristics } \\
\hline Age (year) & $0.57(0.44-0.69)$ & - & - & - & - \\
\hline Age $\geqslant 35$ & $0.55(0.43-0.67)$ & - & - & - & - \\
\hline Nulliparous women & $0.52(0.41-0.64)$ & - & - & - & - \\
\hline Women with a past history of PE/GH & $0.59(0.46-0.71)$ & + or - & 0.23 & 0.94 & $3.7(1.5-9.5)$ \\
\hline Family history of hypertension & $0.61(0.49-0.73)$ & + or - & 0.40 & 0.82 & $2.2(1.3-4.0)$ \\
\hline Current smoking & $0.54(0.42-0.66)$ & - & - & - & - \\
\hline Pre-pregnancy BMI $\left(\mathrm{kg} \mathrm{m}^{-2}\right)$ & $0.66(0.56-0.77)$ & 24.0 & 0.52 & 0.75 & $2.1(1.3-3.3)$ \\
\hline Obesity & $0.59(0.47-0.71)$ & - & - & - & - \\
\hline Chronic hypertension & $0.60(0.48-0.72)$ & + or - & 0.20 & 1.00 & NC \\
\hline MBP at $16-23$ weeks $(\mathrm{mm} \mathrm{Hg})$ & $0.78(0.69-0.88)$ & 91.3 & 0.70 & 0.77 & $3.1(2.1-4.6)$ \\
\hline BN at 20-23 weeks & $0.65(0.52-0.77)$ & + or - & 0.38 & 0.91 & $4.3(2.0-9.1)$ \\
\hline sFIt-1/PIGF ratio at $19-25$ weeks $\left(\left(\mathrm{pg} \mathrm{ml}^{-1}\right) /\left(\mathrm{pg} \mathrm{ml}^{-1}\right)\right)^{\mathrm{a}}$ & $0.75(0.64-0.86)$ & 6.90 & 0.58 & 0.86 & $4.2(2.4-7.4)$ \\
\hline Plasma levels of HSD17B1 at $19-25$ weeks $\left(\mathrm{ng} \mathrm{ml} \mathrm{l}^{-1}\right)^{\mathrm{b}}$ & $0.72(0.62-0.82)$ & 43.0 & 0.86 & 0.56 & $1.9(1.5-2.5)$ \\
\hline \multicolumn{6}{|l|}{ Combination } \\
\hline Using $\mathrm{MBP}$ at $16-23$ weeks and $\mathrm{BN}^{\mathrm{c}}$ & $0.82(0.73-0.91)$ & 0.202 & 0.72 & 0.81 & $3.7(2.4-5.8)$ \\
\hline Using MBP at 16-23 weeks, BN and sFIt-1/PIGF ratio at $19-25$ weeks $^{\mathrm{d}}$ & $0.87(0.79-0.94)$ & 0.241 & 0.73 & 0.85 & $5.0(3.0-8.5)$ \\
\hline Using MBP at 16-23 weeks, BN, sFIt-1/PIGF ratio and HSD17B1 at 19-25 weeks ${ }^{\mathrm{e}}$ & $0.89(0.82-0.96)$ & 0.282 & 0.84 & 0.88 & $7.2(4.0-13)$ \\
\hline
\end{tabular}

Abbreviations: AROC, area under the receiver-operating-characteristic curve; BMI, body mass index; BN, bilateral notching; Cl, confidence interval; GH, gestational hypertension; HSD17B1, hydroxysteroid (17-beta) dehydrogenase 1; LR +, positive likelihood ratio; MBP, mean blood pressure; PE, preeclampsia; PIGF, placental growth factor; SE, sensitivity; sFlt-1, soluble fms-like tyrosine kinase 1; SP, specificity.

aThe level of sFIt-1/PIGF ratio could not be determined in 23 cases.

bThe levels of HSD17B1 could not be determined in 26 cases.

'The combination model using MBP at 16-23 weeks and BN was constructed in 142 cases without missing data.

dThe combination model using MBP at 16-23 weeks, BN and sFIt-1/PIGF ratio at 19-25 weeks was constructed in 129 cases without missing data.

eThe combination model using MBP at 16-23 weeks, BN, sFIt-1/PIGF ratio and HSD17B1 at 19-25 weeks was constructed in 119 cases without missing data. 

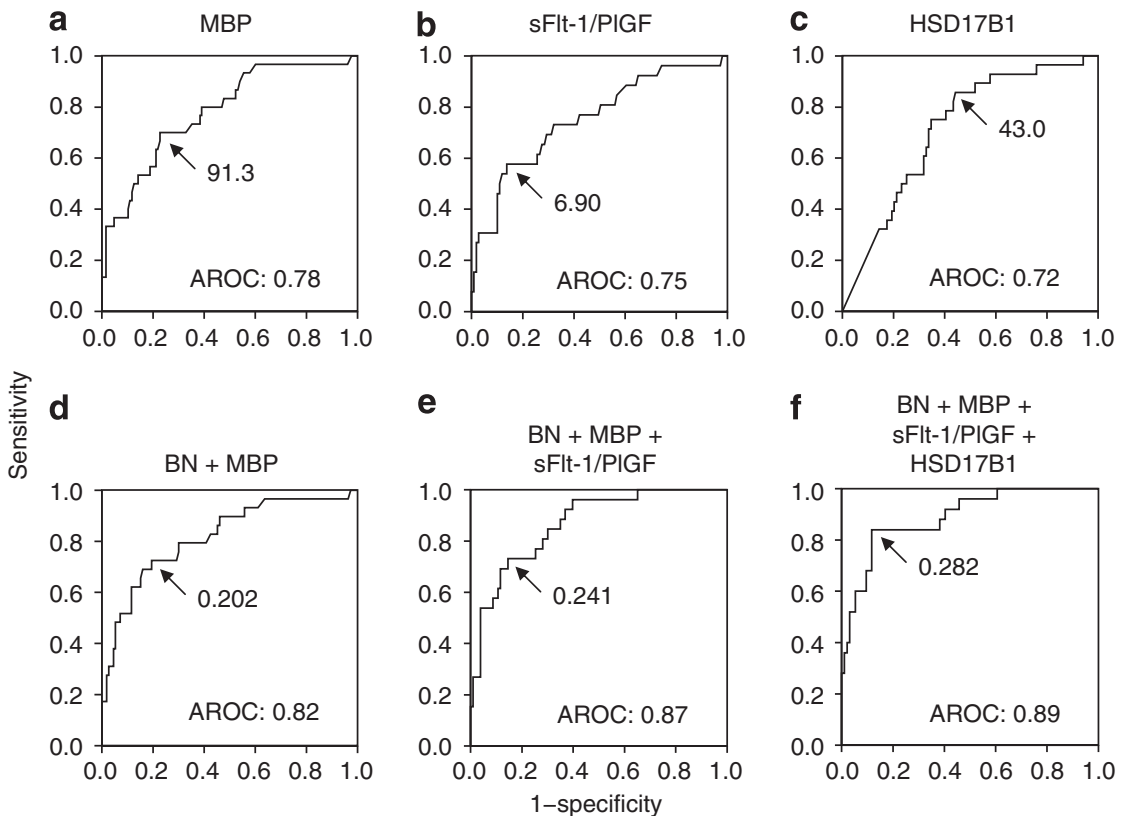

Figure 1 ROC curves of MBP at 16-23 weeks of gestation (a), plasma level of (sFlt-1)/(PIGF) ratio at 19-25 weeks of gestation (b), plasma level of HSD17B1 at 19-25 weeks of gestation (c) for predicting all PE were constructed using 128 normal controls and 30 women who later developed PE. In addition, the ROC curves for predicting all PE by combining BN and MBP (d), by combining BN, MBP and sFIt-1/PIGF ratio (e), and by combining BN, MBP, sFIt-1/PIGF ratio and HSD17B1 (f) were constructed. Arrows show the cutoff values of MBP, sFIt-1/PIGF ratio and the plasma HSD17B1 level in a, b and $\mathbf{c}$; and show the cutoff $P$-values of the combination models in $\mathbf{d}$, e and $\mathbf{f}$.

Table 3 Crude and adjusted odds ratio of risk factors for predicting all PE

\begin{tabular}{|c|c|c|c|c|}
\hline Risk factors & \multicolumn{4}{|c|}{ All preeclamptic women } \\
\hline Past history of GH/PE & $4.6(1.5-14)$ & 0.007 & - & - \\
\hline Family history of hypertension & $3.0(1.3-7.2)$ & 0.011 & - & - \\
\hline $\mathrm{BN}$ & $6.3(2.3-17)$ & $<0.001$ & $7.5(1.9-30)$ & 0.004 \\
\hline $\log _{10}(\mathrm{sFI} I-1 / \mathrm{PIGF})$ at $19-25$ weeks (by 1 increment) & $22(4.4-109)$ & $<0.001$ & $21(2.7-163)$ & 0.003 \\
\hline $\log _{10} \mathrm{HSD} 17 \mathrm{~B} 1$ at $19-25$ weeks (by 1 increment) & $0.54(0.35-0.85)$ & 0.008 & $0.43(0.22-0.85)$ & 0.016 \\
\hline
\end{tabular}

hypertension, a $\mathrm{BN}$ and a sFlt-1/PlGF ratio $\geqslant 6.9$ was significantly higher than that for the normal pregnant group. In addition, in women with EO-PE, the proportion of women with a family history of hypertension and a sFlt-1/PlGF ratio $\geqslant 6.9$ was significantly higher than that for women with LO-PE.

\section{DISCUSSION}

For the first time, we found that MBP, BN, the plasma level of the sFlt-1/PIGF ratio and the plasma level of HSD17B1 in the second trimester are independent risk factors for predicting all PE. The combination of MBP, BN, the sFlt-1/PlGF ratio and HSD17B1 for predicting all $\mathrm{PE}$ yielded a moderate screening performance with an AROC of 0.89 , a sensitivity of 0.84 , a specificity of 0.88 and an LR + (95\% CI) of 7.2, indicating that the combination of maternal factors and plasma biomarkers including a new marker HSD17B1 in the second trimester may be useful for detecting pregnant women who are at high risk for the later occurrence of all PE.

In our previous study, we identified PE-related miRNAs that were aberrantly expressed in preeclamptic placentas and demonstrated that HSD17B1 is post-transcriptionally dysregulated by the PE-related 
Table 4 Comparison of risk factors in normal pregnant women, women with LO-PE and women with EO-PE

\begin{tabular}{lcccccc}
\hline Risk factors & $\begin{array}{c}\text { Normal pregnant women } \\
(N P)(n=128)\end{array}$ & $\begin{array}{c}\text { Women with } \\
\text { LO-PE }(\mathrm{n}=24)\end{array}$ & $\begin{array}{c}\text { Women with } \\
\text { EO-PE }(\mathrm{n}=6)\end{array}$ & $\begin{array}{c}\text { Numbers of } \\
\text { missing values }\end{array}$ & P-value & Significant pair(s) \\
\hline Past history of GH/PE (\%) & $8(6.3)$ & $5(21)$ & $2(6)$ & 0 & 0.010 & NP vs. LO-PE \\
Family history of hypertension (\%) & $23(18)$ & $7(29)$ & $5(83)$ & 0 & $<0.001$ & NP vs. EO-PE, LO- vs. EO-PE \\
Pre-pregnancy BMI $\geqslant 24(\%)$ & $32(25)$ & $12 / 23(52)$ & $3(50)$ & 1 & 0.018 & NP vs. LO-PE \\
MBP $\geqslant 91.3(\%)$ & $29(23)$ & $18(75)$ & $3(50)$ & 0 & $<0.001$ & NP vs. LO-PE \\
BN & $10 / 113(8.8)$ & $7 / 23(30)$ & $4(67)$ & 26 & $<0.001$ & NP vs. LO-PE, NP vs. EO-PE \\
sFIt-1/PIGF $\geqslant 6.9(\%)$ & $15 / 109(14)$ & $9 / 20(45)$ & $6 / 6(100)$ & 23 & $<0.001$ & all pairs \\
HSD17B1 <43 $\%)$ & $46 / 104(44)$ & $20 / 23(87)$ & $4 / 5(80)$ & 26 & $<0.001$ & NP vs. LO-PE \\
\hline
\end{tabular}

Abbreviations: BMI, body mass index; BN, bilateral notching; EO-PE, women with early-onset PE; GH, gestational hypertension; HSD17B1, hydroxysteroid (17-beta) dehydrogenase 1; LO-PE, women with late-onset PE; MBP, mean blood pressure; NP, normal pregnant women; PE, preeclampsia; PIGF, placental growth factor; sFlt-1, soluble fms-like tyrosine kinase 1.

We defined PE with onset earlier than 32 weeks of gestation as EO-PE, and onset at 32 weeks of gestation or later as LO-PE.

miRNAs, $m i R-210$ and $m i R-518 c .{ }^{8}$ Moreover, a prospective cohort study of plasma HSD17B1 in pregnant women before and after PE onset showed a significant reduction of plasma levels of HSD17B1. ${ }^{8}$ The plasma levels of HSD17B1 were significantly decreased at 20-23 weeks and 27-30 weeks before the onset of LO-PE or term PE, as well as all $\mathrm{PE}$, suggesting that decreases in plasma HSD17B1 levels are potentially new predictive markers for LO-PE or term PE. ${ }^{8}$ Our current study revealed that the plasma level of HSD17B1 at 19-25 weeks of gestation was an independent risk factor for all PE after adjusting for the effects of blood pressure levels, $\mathrm{BN}$ and the plasma levels of the sFlt-1/PlGF ratio. The addition of HSD17B1 to the multivariate model, including $\mathrm{MBP}, \mathrm{BN}$ and the sFlt-1/PlGF ratio together, also changed LR + from 5.0 to 7.2 , suggesting that HSD17B1 might have a different mechanism for the genesis of PE from angiogenesis-related factors. Actually, the Spearman's correlation between the sFlt-1/PlGF ratio and HSD17B1 was not significant, supporting our hypothesis. The serum level of the sFlt-1/PlGF ratio was related to the genesis of EO-PE or PE with onset at $<36$ weeks of gestation; ${ }^{3}$ however, the plasma levels of the sFlt-1/PlGF ratio at 22-26 weeks did not significantly increase in women who developed PE qafter 34 weeks of gestation; ${ }^{15}$ and the serum sFlt-1/PlGF ratio at 21-32 weeks did not greatly increase in preeclamptic women who delivered after 37 weeks (term PE). ${ }^{7}$ These results indicate that the abnormalities of angiogenesis-related factors are more related to the genesis of the EO-PE. In contrast, the proportion of women having a level of HSD17B1 $<43 \mathrm{ngml}^{-1}$ at $19-25$ weeks was almost twice as high in both women with LO-PE and women with EO-PE as in normal pregnant women, although the difference between women with EO-PE and normal pregnant women was not significant, possibly due to the small sample size. The plasma levels of HSD17B1 after the onset of PE were significantly decreased in both EO-PE and LO-PE. ${ }^{8}$ These results suggest that the decrease of HSD17B1 might be related to essential basic patho-physiological changes by which all PE are affected.

In our study, the LR + using only HSD17B1 increased from 1.9 to 7.2 in the prediction model involving HSD17B1, MBP, BN and the sFlt-1/PlGF ratio, indicating that the combination of several markers is useful for predicting all $\mathrm{PE}$ in the second trimester. The $\mathrm{LR}+\mathrm{s}$ using a single variable (MBP, BN, sFlt-1/PlGF ratio or HSD17B1) were all $<5$, indicating that the prediction of all $\mathrm{PE}$ in the second trimester by using a single variable is not clinically useful. In our previous report, we demonstrated that the multivariate approach including a previous history of GH/PE, MBP at 16-23 weeks and serum levels of soluble endoglin yielded an LR + of 12 for predicting all PE. ${ }^{3}$ However, in the current study, the LR + using four variables, not including soluble endoglin, was $<10$; therefore, the addition of the circulating level of soluble endoglin to the current multivariate model might improve the screening performance in the current model.

Growing evidence has verified that angiogenesis imbalance in pregnancy is implicated in the pathophyisology and pathogenesis of pregnancy-induced hypertension. ${ }^{3,7,9,15-25}$ In this study, we also revealed that a new and automated electrochemiluminescence immunoassay for plasma sFlt-1 and PlGF levels at 19-25 weeks of gestation had potential predictive power for predicting all PE. In our current study, the LR + of the plasma level of the sFlt-1/PlGF ratio at 19-25 weeks for predicting all PE was 4.2, whereas in the previous study using an ELISA kit (R\&D Systems, Minneapolis, MN, USA), the LR + of the serum level of the sFlt-1/PlGF ratio at 19-25 weeks for predicting all PE was 4.7 (Ohkuchi et al. ${ }^{3}$ ). Thus, the screening performance of our new assay for predicting all PE in the second trimester was almost identical to the standard ELISA assay. Our previous study also revealed that the serum level of the sFlt-1/PlGF ratio at 19-25 weeks was a moderate predictor of EO-PE with an LR + of 9.3 (Ohkuchi et al. ${ }^{3}$ ). In the current study, the LR $+\mathrm{s}$ of the plasma level of the sFlt-1/PlGF ratio at 19-25 weeks for predicting EO-PE and LO-PE were 7.3 and 3.3, respectively (data not shown). These results suggested that the measurement of the circulating level of the sFlt-1/ PlGF ratio in the second trimester mainly serves to predict the pregnant women at high risk for EO-PE, but partly to predict pregnant women at high risk for LO-PE.

The numbers of PE patients were relatively small for sub-analyses to evaluate the independent risk factors for EO-PE or LO-PE. When we constructed multivariate logistic models including MBP, BN, the sFlt-1/PlGF ratio and HSD17B1 for predicting LO-PE, the adjusted OR $(95 \% \mathrm{CI})$ of HSD17B1 $<43 \mathrm{ng} \mathrm{ml}^{-1}$ was $3.5(0.24-1.03)$ $(P=0.060)$ (data not shown), suggesting that HSD17B1 might be an independent risk factor for LO-PE, if more subjects are analyzed in the future.

In a previous study, we demonstrated that the relative expression levels of HSD17B1 mRNA and protein in preeclamptic placentas were significantly decreased than those in normal placentas, ${ }^{8}$ supporting previous data on the pronounced downregulation of HSD17B1 gene in preeclamptic placenta demonstrated by comprehensive microarray analysis. ${ }^{26}$ In addition, in the current study, we revealed that the decrease of plasma levels of HSD17B1 may precede the clinical onset of $\mathrm{PE}$ in the second trimester, without relating to the abnormalities of angiogenesis-related factors. As HSD17B1 is a steroidogenetic enzyme 
catalyzing the conversion of estrone to $17 \beta$-estradiol, ${ }^{27,28}$ the decrease of $17 \beta$-estradiol might be involved in the genesis of PE. $17 \beta$-Estradiol is converted to hydroxyestradiol by the action of cytochrome $\mathrm{P} 450$, and hydroxyestradiol is converted to 2-methoxyestradiol by the action of catechol-O-methyltransferase (COMT)..$^{29,30}$ The decrease of 2-methoxyestradiol by the deficient of COMT induced the PE-like phenotype via the decrease of 2-methoxyestradiol in mice, ${ }^{29,30}$ indicating that some derivatives of estradiol might be implicated in the genesis of PE. Several studies of polymorphisms of estrogen receptor gene also suggested the possible implication of estrogen for the genesis of PE. ${ }^{31-34}$ Molvarec et al. ${ }^{34}$ speculated that the reduced estrogen receptor alpha (ESR1) expression in pregnant women associated with certain ESR1 gene polymorphisms might disturb the ESR1-mediated effects of estrogen, leading to a relative estrogen-deficient state. Estrogen has several effects on vascular tissues and systemic effects, such as vasodilatory effects, acceleration of re-endothelialization after vascular injury, inhibition of the vascular injury response, changes in lipid profiles, alterations in coagulation and fibrinolytic systems and antioxidant effects; therefore, the relative estrogen deficit might lead to vasoconstriction in the systemic and uteroplacental circulation in pregnant women and may influence the risks of PE. Taken together, relative estrogen deficiency might generate PE via vasoconstriction in the systemic and uteroplacental circulation in pregnant human beings and mice. Indeed, in our data, a weak negative correlation between the plasma level of HSD17B1 and MBP in the second trimester was observed in pregnant women $(R=-0.266, P=0.002)$ (data not shown), supporting the hypothesis.

One key limitation is the small sample size in our study. Only 30 women with PE, especially only six patients of EO-PE, in 1724 pregnant cohorts could have been biased by sampling error. Therefore, another larger prospective cohort study should be planned to evaluate our hypothesis that reducing plasma levels of HSD17B1 is a potential independent prognostic factor for not only EO-PE but also LO-PE.

In conclusion, $\mathrm{MBP}, \mathrm{BN}$, the plasma level of the sFlt-1/PlGF ratio and the plasma level of HSD17B1 were independent risk factors for all $\mathrm{PE}$. The combination of the four markers in the second trimester yielded moderate predictive power for all PE. Moreover, the plasma levels of HSD17B1 in the second trimester were decreased in pregnant women with a later occurrence of LO-PE or term PE. ${ }^{8}$ Therefore, the measurement of the plasma level of HSD17B1, in addition to previously known risk factors, may improve the detection of pregnant women at high risk of PE in the second trimester. The development of a commercially available ELISA kit for HSD17B1 is a matter of great urgency for clinical introduction for the measurement of the plasma level of HSD17B1.

\section{CONFLICT OF INTEREST}

The authors declare no conflict of interest.

\section{ACKNOWLEDGEMENTS}

This work was supported by Grants-in-Aid (18591809 and 24592482 to AO, 20390437 and 24390383 to TT) and the 'Research Core' Project for Private University: Matching Fund Subsidy from the Ministry of Education, Culture, Sports, Science and Technology in Japan, a research grant from the Japan Society for the Study of Hypertension in Pregnancy, and a research grant (3-2417-011 to SM) from the Japan Association of Obstetricians and Gynecologists Ogyaa Donation Foundation. We greatly thank Roche Diagnostics for the kind gifts of the Elecsys sFlt- 1 and Elecsys PlGF, and for the cooperation with measurements of sFlt-1 and PlGF.
1 Duckitt K, Harrington D. Risk factors for pre-eclampsia at antenatal booking: systematic review of controlled studies. BMJ 2005; 330: 565.

2 Hernández-Díaz S, Toh S, Cnattingius S. Risk of pre-eclampsia in first and subsequent pregnancies: prospective cohort study. BMJ 2009; 338: b2255.

3 Ohkuchi A, Hirashima C, Matsubara S, Takahashi K, Matsuda Y, Suzuki M. Threshold of soluble fms-like tyrosine kinase $1 /$ placental growth factor ratio for the imminent onset of preeclampsia. Hypertension 2011; 58: 859-866.

4 Qiu C, Williams MA, Leisenring WM, Sorensen TK, Frederick 10, Dempsey JC, Luthy DA. Family history of hypertension and type 2 diabetes in relation to preeclampsia risk. Hypertension 2003; 41: 408-413.

5 Ohkuchi A, Iwasaki R, Suzuki H, Hirashima C, Takahashi K, Usui R, Matsubara S, Minakami H, Suzuki M. Normal and high-normal blood pressures, but not body mass index, are risk factors for the subsequent occurrence of both preeclampsia and gestational hypertension: a retrospective cohort study. Hypertens Res 2006; 29: $161-167$.

6 Cnossen JS, Morris RK, ter Riet G, Mol BW, van der Post JA, Coomarasamy A, Zwinderman AH, Robson SC, Bindels PJ, Kleijnen J, Khan KS. Use of uterine artery Doppler ultrasonography to predict pre-eclampsia and intrauterine growth restriction: $a$ systematic review and bivariable meta-analysis. CMAJ 2008; 178: 701-711.

7 Levine RJ, Lam C, Qian C, Yu KF, Maynard SE, Sachs BP, Sibai BM, Epstein FH, Romero R, Thadhani R, Karumanchi SA; CPEP Study Group. Soluble endoglin and other circulating antiangiogenic factors in preeclampsia. N Engl J Med 2006; 355: 992-1005.

8 Ishibashi O, Ohkuchi A, Ali MM, Kurashina R, Luo SS, Ishikawa T, Takizawa T, Hirashima C, Takahashi K, Migita M, Ishikawa G, Yoneyama K, Asakura H, Izumi A, Matsubara S, Takeshita T, Takizawa T. Hydroxysteroid (17- $\beta$ ) dehydrogenase 1 is dysregulated by Mir-210 and Mir-518c that are aberrantly expressed in preeclamptic placentas: a novel marker for predicting preeclampsia. Hypertension 2012; 59: 265-273.

9 Ohkuchi A, Hirashima C, Suzuki H, Takahashi K, Yoshida M, Matsubara S, Suzuki M. Evaluation of a new and automated electrochemiluminescence immunoassay for plasma sFIt-1 and PIGF levels in women with preeclampsia. Hypertens Res 2010; 33: 422-427.

10 Sato K. A proposal for a new definition and classification of "pregnancy induced hypertension (PIH)" (2004). In: Japan Society for the Study of Toxemia of Pregnancy, ed. Historical Perspective of Study of Pregnancy-Induced Hypertension in Japan. Medical View Co, Tokyo, Japan, 2005, pp 54-87.

11 von Dadelszen P, Magee LA, Roberts JM. Subclassification of preeclampsia. Hypertens Pregnancy 2003; 22: 143-148.

12 Raymond D, Peterson E. A critical review of early-onset and late-onset preeclampsia. Obstet Gynecol Surv 2011; 66: 497-506.

13 Soto E, Romero R, Kusanovic JP, Ogge G, Hussein Y, Yeo L, Hassan SS, Kim CJ, Chaiworapongsa T. Late-onset preeclampsia is associated with an imbalance of angiogenic and anti-angiogenic factors in patients with and without placental lesions consistent with maternal underperfusion. J Matern Fetal Neonatal Med 2012; 25: 498-507.

14 Ogawa Y, Iwamura T, Kuriya N, Kuriya N, Nishida H, Takeuchi H, Yakada M, Itabashi K, Imura S, Isobe K. Birth size standards by gestational age for Japanese neonates. Acta Neonatol Jpn 1998; 34: 624-632.

15 Moore Simas TA, Crawford SL, Solitro MJ, Frost SC, Meyer BA, Maynard SE. Angiogenic factors for the prediction of preeclampsia in high-risk women. Am J Obstet Gynecol 2007; 197(244): 244e1-244e8.

16 Maynard SE, Min JY, Merchan J, Lim KH, Li J, Mondal S, Libermann TA, Morgan JP, Sellke FW, Stillman IE, Epstein FH, Sukhatme VP, Karumanchi SA. Excess placental soluble fms-like tyrosine kinase 1 (sFlt1) may contribute to endothelial dysfunction, hypertension, and proteinuria in preeclampsia. J Clin Invest 2003; 111: 649-658.

17 Levine RJ, Maynard SE, Qian C, Lim KH, England LJ, Yu KF, Schisterman EF, Thadhani R, Sachs BP, Epstein FH, Sibai BM, Sukhatme VP, Karumanchi SA. Circulating angiogenic factors and the risk of preeclampsia. N Engl J Med 2004; 350: 672-683.

18 Hirashima C, Ohkuchi A, Arai F, Takahashi K, Suzuki H, Watanabe T, Kario K, Matsubara S, Suzuki M. Establishing reference values for both total soluble Fms-like tyrosine kinase 1 and free placental growth factor in pregnant women. Hypertens Res 2005; 28: 727-732.

19 Venkatesha S, Toporsian M, Lam C, Hanai J, Mammoto T, Kim YM, Bdolah Y, Lim KH, Yuan HT, Libermann TA, Stillman IE, Roberts D, D'Amore PA, Epstein FH, Sellke FW, Romero R, Sukhatme VP, Letarte M, Karumanchi SA. Soluble endoglin contributes to the pathogenesis of preeclampsia. Nat Med 2006; 12: 642-649.

20 Ohkuchi A, Hirashima C, Matsubara S, Suzuki H, Takahashi K, Arai F, Watanabe T, Kario K, Suzuki M. Alterations in placental growth factor levels before and after the onset of preeclampsia are more pronounced in women with early onset severe preeclampsia. Hypertens Res 2007; 30: 151-159.

21 Hirashima C, Ohkuchi A, Matsubara S, Suzuki H, Takahashi K, Usui R, Suzuki M. Alteration of serum soluble endoglin levels after the onset of preeclampsia is more pronounced in women with early-onset. Hypertens Res 2008; 31: 1541-1548.

22 Suzuki H, Ohkuchi A, Matsubara S, Takei Y, Murakami M, Shibuya M, Suzuki M, Sato $Y$. Effect of recombinant placental growth factor 2 on hypertension induced by fulllength mouse soluble fms-like tyrosine kinase 1 adenoviral vector in pregnant mice. Hypertension 2009; 54: 1129-1135. 
23 Kulkarni AV, Mehendale SS, Yadav HR, Kilari AS, Taralekar VS, Joshi SR. Circulating angiogenic factors and their association with birth outcomes in preeclampsia. Hypertens Res 2010; 33: 561-567.

24 Molvarec A, Szarka A, Walentin S, Szucs E, Nagy B, Rigó Jr J. Circulating angiogenic factors determined by electrochemiluminescence immunoassay in relation to the clinical features and laboratory parameters in women with pre-eclampsia. Hypertens Res 2010; 33: 892-898.

25 Hirashima C, Ohkuchi A, Takahashi K, Suzuki H, Yoshida M, Ohmaru T, Eguchi K, Ariga $\mathrm{H}$, Matsubara S, Suzuki M. Gestational hypertension as a subclinical preeclampsia in view of serum levels of angiogenesis-related factors. Hypertens Res 2011; 34: 212-217.

26 Winn VD, Gormley M, Paquet AC, Kjaer-Sorensen K, Kramer A, Rumer KK, HaimovKochman R, Yeh RF, Overgaard MT, Varki A, Oxvig C, Fisher SJ. Severe preeclampsiarelated changes in gene expression at the maternal-fetal interface include sialic acidbinding immunoglobulin-like lectin-6 and pappalysin-2. Endocrinology 2009; 150 452-462.

27 Peltoketo $\mathrm{H}$, Isomaa V, Poutanen M, Vihko R. Expression and regulation of 17 beta-hydroxysteroid dehydrogenase type 1. J Endocrinol 1996: 150(Suppl): S21-S30.

28 Peltoketo H, Vihko P, Vihko R. Regulation of estrogen action: role of 17 betahydroxysteroid dehydrogenases. Vitam Horm 1999; 55: 353-398.
29 Kanasaki K, Palmsten K, Sugimoto H, Ahmad S, Hamano Y, Xie L, Parry S Augustin HG, Gattone VH, Folkman J, Strauss JF, Kalluri R. Deficiency in catechol0-methyltransferase and 2-methoxyoestradiol is associated with pre-eclampsia. Nature 2008; 453: 1117-1121.

30 Shenoy V, Kanasaki K, Kalluri R. Pre-eclampsia: connecting angiogenic and metabolic pathways. Trends Endocrinol Metab 2010; 21: 529-536.

31 Malamitsi-Puchner A, Tziotis J, Evangelopoulos D, Fountas L, Vlachos G, Creatsas G, Sekeris CE, Moutsatsou P. Gene analysis of the N-terminal region of the estrogen receptor alpha in preeclampsia. Steroids 2001; 66: 695-700.

32 Tempfer CB, Jirecek S, Riener EK, Zeisler H, Denschlag D, Hefler L, Husslein PW. Polymorphisms of thrombophilic and vasoactive genes and severe preeclampsia: a pilot study. J Soc Gynecol Investig 2004; 11: 227-231.

33 Maruyama A, Nakayama T, Sato N, Mizutani Y, Furuya K, Yamamoto T. Association study using single nucleotide polymorphisms in the estrogen receptor beta (ESR2) gene for preeclampsia. Hypertens Res 2004; 27: 903-909.

34 Molvarec A, Vér A, Fekete A, Rosta K, Derzbach L, Derzsy Z, Karádi I, Rigó Jr J. Association between estrogen receptor alpha (ESR1) gene polymorphisms and severe preeclampsia. Hypertens Res 2007; 30: 205-211. 